\title{
Diffusive shock acceleration and radio emission from shell-type SNRs
}

\author{
A. I. Asvarov \\ Institute of Physics, Baku AZ1143, Azerbaijan; email: asvarov@physics.ab.az
}

\begin{abstract}
Diffusive shock acceleration (DSA) in test-particle approximation is used for explaining the radio emission from shell-type SNRs.
\end{abstract}

Keywords. supernova remnants, radiation mechanisms: nonthermal, acceleration of particles

Almost all known SNRs are sources of synchrotron radio emission. Naturally, much richer observational information on the SNRs is available in the radio range that can be used for explaining the origin of high energy electrons and magnetic fields responsible for the synchrotron emission. DSA is the most suitable mechanism for explaining the origin of radio-emitting electrons. But in the theory of DSA some principal aspects remain unclear. Modeling of the SNR radio evolution by using the most reliable and common theoretical results of DSA and comparison with observational data can be used for resolving these questions. A model of radio emission of shell-type SNRs using test-particle DSA has been developed in a recent paper by the author (Asvarov 2006). It is based on the assumptions that electrons are injected into the mechanism directly from the high energy tail of the downstream Maxwellian distribution function, and the magnetic field is compressed at the typical interstellar field shock .

The model predicts that the radio surface brightness $\left(\Sigma_{R}\right)$ evolves with diameter as $\propto D^{-(0.3 \div 0.5)}$ while the bounding shock wave is strong (Mach number $\mathcal{M} \geqslant 10$ ) followed by steep decrease (steeper than $\propto D^{-4.5}$ ) for $\mathcal{M}<10$. This result is in good agreement with the conclusion of Berkhuijsen (1986) about the $\Sigma_{\mathrm{R}}-D$ relations. The dependence of the nearly constant $\Sigma_{\mathrm{R}}$ on the environmental and initial parameters is expressed as $\Sigma_{\mathrm{R}} \propto n_{\mathrm{e} 0}^{2 / 3} B_{0}^{3 / 2} E_{\mathrm{SN}}^{1 / 2} M_{\mathrm{ej}}^{-1 / 6}$. The shape of the dependence of $\Sigma_{\mathrm{R}}$ on $D$ and other initial and environmental parameters greatly reduce the usefulness of $\Sigma_{\mathrm{R}}-D$ relations as a tool for determining the distances to SNRs. Our model easily explains both very large diameter radio sources such as Galactic Loops and candidates for Hypernova radio remnants and small size radio sources as the remnant of Nova Persei 1901. The model predicts no radio emission from the radiative SNRs and the existence of radio quiet but relatively active SNRs is possible.

From the comparison of the model results with the statistics of evolved shell-type SNRs we were able to estimate the fraction of electrons accelerated from the thermal pool as in the range $(3 \div 11) \times 10^{-4}$. If acceleration takes place from a Maxwellian distribution function then the corresponding injection momentum is estimated as $p_{\mathrm{inj}} \simeq(2.7 \div 3) \cdot p_{\mathrm{th}}$. If the Galactic Loops are actually SNRs then from our models it follows that DSA still acts at very weak shocks (say, with $\mathcal{M} \leqslant 3$ ).

\section{References}

Asvarov, A. I. 2006, A\&AA, 459, 519

Berkhuijsen, E. M. 1986, A\&\&A 166, 257 